\title{
Utilización de las consultas de pediatría de Atención Primaria y morbilidad en la ciudad de Toledo. Comparación entre una cohorte de hijos de españoles y otra de hijos de inmigrantes
}

\author{
N. Pérez Villaverde, M. Soto García ${ }^{1}$, L. Rivilla Marugán², \\ S. CAstillo Portales'1, N. Campos Campos ${ }^{3}$, N. Marañón HenRich \\ Médicos Especialistas en Medicina Familiar y Comunitaria. \\ Centro de Salud de Palomarejesentro de Salud de \\ Los Yébenes.Centro de Salud Nobleją̧entro de Salud de Polán. Toledo
}

\section{RESUMEN}

Objetivos: conocer el uso de consultas de pedia tría de Atención Primaria y morbilidad en hijos de inmigrantes (HI) comparándolos con hijos de po blación autóctona (HA). trol.

Diseño: estudio observacional con grupo con -

Material y métodos: muestras de 147 hijos de inmigrantes (HI) y 147 hijos de la población autóc tona (HA) apareados por edad y sexo, de los 4 Centros de Salud de Toledo, recogiéndose de las historias clínicas datos demográficos, inicio y du ración de lactancia materna, asistencia a consultas programadas, situación calendario vacunal, núme ro, motivos de consulta y pruebas complementarias solicitadas en un año (junio 2000-junio 2001).

Resultados: el número medio de consultas/año fue similar en ambos grupos, 2,12 (DE 2,43) y 2,56 (DE 2,96) en HI y HA respectivamente. El $37,41 \%$ de HI faltó en alguna ocasión a consulta progra mada, frente al $17 \%$ de HA $(p<0,05)$. El motivo de consulta más frecuente en ambos grupos fue infec ción respiratoria de vías altas $(63,37 \%$ en $H I$ vs $73,79 \%$ en HA). Las gastroenteritis agudas y las otitis fueron menos frecuentes en HI que en HA $(9,89$ vs $20,39 \% ; 10,88$ vs $23,3 \%$ respectivamente, $p<0,05)$, lo contrario que los dolores abdominales inespecíficos $(9,9 \%$ en $H I$ vs $1,94 \%$ en $H A$, $p<0,05)$, el resto de motivos de consulta fue similar en ambos grupos. Se solicitaron 39 pruebas complementarias en HI y 29 en HA, destacando el
Use of pediatric Primary Health Care consultation morbility in Toledo. Comparation between a Spa nish children cohort and an inmigration one

\section{ABSTRACT}

Objectives: to study the use of pediatric Primary Health Care consultations by inmigrants children (IC) and the morbility of this group, comparing to native children $(N C)$.

Design: observational study with control group.

Material and methods: two samples of 147 IC and 147 NC matched by sex and age, from the 4 Primary Health Care Centres in Toledo. From the histories were collected dates about demography, beginning and lasting of breast feeding, atten dance to programmed visits, vaccinal situation, demanding's number and causes, and laboratory tests required along one year (June 2000-June 2001).

Results: the mean number of demands by year was similar in both groups IC and NC, 2.12 (SD 2.43 ) and $2.56(S D 2.96)$ respectively. $37.21 \%$ of IC failed, at least once, to a programmed visit, comparing to $17 \%$ of $N C(p<0.05)$. The most frequent demanding cause was upper respiratory tract infection in both groups $(63.37 \%$ at IC vs $73.79 \%$ at NC). Acute gastroenteritis and otitis were less frequent among IC than NC $(9.98 \mathrm{vs}$ $20.39 \%$; 10.88 vs $23.3 \%$ respectively, $p<0.05$ ). The opposite happened with inespecific abdomi nal pain (9.9 at IC vs $1.94 \%$ at NC, $p<0.05$ ). The rest of demanding causes were similar in both groups. Thirty-nine laboratory tests were asked for IC group and 29 for NC, being remarkable Mantoux (four times for IC and none for NC). 
Mantoux, solicitado en 4 ocasiones en HI y ningu na en HA. La duración de la lactancia natural fue mayor en inmigrantes, 4,59 meses (DE 3,25), que en autóctonos, 3,52 meses (DE 2,08), $p<0,05$.

Conclusiones: los HI se comportan de forma muy similar a los HA en cuanto al número de con sultas anuales y a las patologías más frecuente mente observadas. Sin embargo, la diferencia en contrada en algunas patologías, el alto incumplimiento a consultas programadas y la mayor duración de la lactancia materna nos recuerda que estamos ante una población con aún diferen cias socioculturales.

Palabras clave: Inmigrantes. Atención Prima ria. Morbilidad. Pediatría. Utilización.
Breast feeding lasting was longer at IC group, 4.59 months (SD 3.25) than at NC group, 3.52 months (SD 2.08), $p<0.05$.

Conclusions: IC behaviour is very similar to $N C$, regarding number of annual demandings and more frequent pathologies. However there are things that reminds us they are a population that still have some particularity: differences found among some pathologies, the high non-attendance rate to programmed visits at IC group, and the lon ger lasting of breast feeding at this group, are clear examples.

Key words: Inmigrants. Primary Health Care. Morbility. Pediatric. Use.

\section{INTRODUCCIÓN}

El aumento progresivo de los flujos migratorios de personas procedentes de países en vías de desarrollo hacia los países industrializados está cambiando a un ritmo vertiginoso el entramado social de éstos. En España, asistimos desde 1985 a un aumento de esta población inmigrante, que actualmente se calcula que podría representar el $0,6 \%$ de la población total, muy por debajo aún del resto de la Unión Europea donde esta cifra llega al $10 \%{ }^{1}$.

El médico de familia y pediatra de Atención Primaria ve reflejado este hecho en la asistencia a una población étnicamente más plural y, en consecuencia, con problemas y dificultades hasta ahora ajenos a la práctica médica habitual en nuestro entor$\mathrm{no}^{2-6}$.

Además, debido no sólo al propio proceso migratorio, sino también a las condiciones de vida en los países de acogida, la salud de la población inmigrante puede sufrir problemas añadidos que influyan en la utilización de los servicios sanitarios ${ }^{3-5,7,8}$.

Por ello creemos conveniente tener un mayor conocimiento de esta realidad y su repercusión en las consultas de Atención Primaria. Surge así este estudio, con el objetivo de conocer el uso que hacen los niños hijos de inmigrantes (HI) de las consultas de pediatría en el ámbito de la Atención Primaria de la ciudad de Toledo y comparar su morbilidad con la de los niños hijos de la población autóctona (HA).

\section{MATERIAL Y MÉTODOS}

Se trata de un estudio observacional con grupo control realizado en los cuatro Centros de Salud que atienden a la población de la ciudad de Toledo.
Los datos del estudio se obtuvieron tras la revisión de las historias clínicas de niños y niñas de 0 a 14 años, de las siete consultas de pediatría de dichos Centros.

A través de los listados generales de usuarios existentes en cada Centro de Salud y con la colaboración de los pediatras y personal de enfermería de los mismos se obtuvo una relación de todos los HI de países extracomunitarios. Se excluyeron aquéllos que no tenían historia clínica y los que ésta había sido abierta en el periodo de observación. A continuación, de forma aleatoria, se obtuvo otra muestra de niños HA apareados por edad y sexo respecto a la anterior.

Las variables recogidas de las historias clínicas incluían datos demográficos (edad, sexo, país de origen de los padres, lugar de nacimiento de los niños), datos referentes a la gestación y parto (semana de gestación al nacer, tipo de parto, peso e ingreso del niño tras el nacimiento), lactancia materna o artificial, la constancia de la situación del calendario vacunal en las historia, el cumplimiento de las visitas programadas, así como el número de veces que acudieron a consulta a deman$\mathrm{da}$, el motivo de las mismas y las pruebas complementarias solicitadas a lo largo de un año (junio del 2000 a junio del 2001).

Las variables países de origen y nacimiento de los niños se estratificaron en Ecuador, Colombia, China, Rumanía y España por presentar un mayor número de casos, agrupando el resto de los países por áreas geográficas (Magreb, África negra y resto de Latinoamérica).

Para el análisis estadístico de los datos se utilizó el programa informático SPSS 9.0, empleándose los tests de Chi cuadrado y ANOVA para la comparación de porcentajes y medias respectivamente. 


\section{RESULTADOS}

Se obtuvo un listado de 159 niños hijos de inmigrantes, no existiendo historia clínica abierta en 5 de ellos y en 7 había sido abierta en el periodo de observación, por lo que la muestra a estudio fue de 147 historias de niños HI, de los cuales 70 $(47,62 \%)$ eran niños y $77(52,38 \%)$ niñas. La edad media fue de 5,5 años (DE 3,6).

La nacionalidad de los padres y el lugar de nacimiento de los niños se recoge en la figura 1.

Los factores en relación con la gestación y parto se reflejan en la tabla I, no encontrando diferencias estadísticamente significativas entre ambas poblaciones en cuanto al "tipo de parto" y al "ingreso del niño tras el nacimiento"; no siendo valorable la significación estadística en las variables "semana de gestación al nacer" y "peso del niño tras el nacimiento" puesto que el número de casos encontrados en alguna de las categorías es muy pequeño. En un $35 \%$ de las historias de los HI no constaba alguno de estos datos, mientras que esta ausencia se objetivó en un $15 \%$ de las historias de HA. Este hecho podría suponer un sesgo al comparar estas variables.

Las diferencias encontradas en los dos grupos a estudio, en relación al tipo de lactancia, constancia de la situación del calendario vacunal en las historias clínicas y asistencia a consultas programadas se muestran en la tabla II y en la figura 2. En 48 $(32,65 \%)$ historias de HI no constaba el tipo de lactancia recibida, versus $14(9,54 \%)$ historias de HA.

De los niños que lactaron la media fue de 4,59 meses (DE 3,25) en los HI, frente a 3,52 meses (DE 2,08) en los HA, siendo esta diferencia estadísticamente significativa $(\mathrm{p}<0,05)$.

El número medio de consulta a lo largo del año de observación no presentó diferencias significati- vas siendo de 2,12 (DE 2,43) y 2,56 (DE 2,96) para las dos poblaciones, HI y HA respectivamente.

Las causas más frecuentes de consulta en ambas muestras fueron las infecciones respiratorias de vías altas. Sólo se encontraron diferencias significativas $(p<0,05)$ en las otitis, gastroenteritis aguda y dolor abdominal inespecífico. Los motivos de consulta aparecen en la figura 3.

Se solicitaron 39 y 29 pruebas complementarias en HI y HA respectivamente, siendo similares en ambas muestras salvo la petición de Mantoux a 4 niños HI y ninguno a HA $(\mathrm{p}<0,05)$. Los resultados de esta prueba fueron negativos en todos los casos (Fig. 4).

\section{CONCLUSIONES}

La multiculturalidad de la sociedad actual, consecuencia de la inmigración, supone un nuevo reto para los profesionales de la Atención Primaria pues tienen que intervenir en situaciones hasta ahora desconocidas $^{1,9,10}$.

Si bien distintos estudios analizan los motivos de consulta y patologías de la población inmigrante ${ }^{8,9,14}$, no hemos encontrado estudios comparativos realizados en España que muestren si existen diferencias entre dicha población y la autóctona, por lo que creemos que nuestro estudio presenta un aspecto novedoso en este tema, al comparar ambas poblaciones en edad pediátrica, grupo, por otra parte, poco estudiado.

La obtención de información sobre el estado de salud de la población inmigrante, así como la utilización de los servicios sanitarios, es difícil por las características propias de estos colectivos como por la deficiente recogida de información ${ }^{5}$. Este hecho también ha sido constatado en nuestro trabajo,

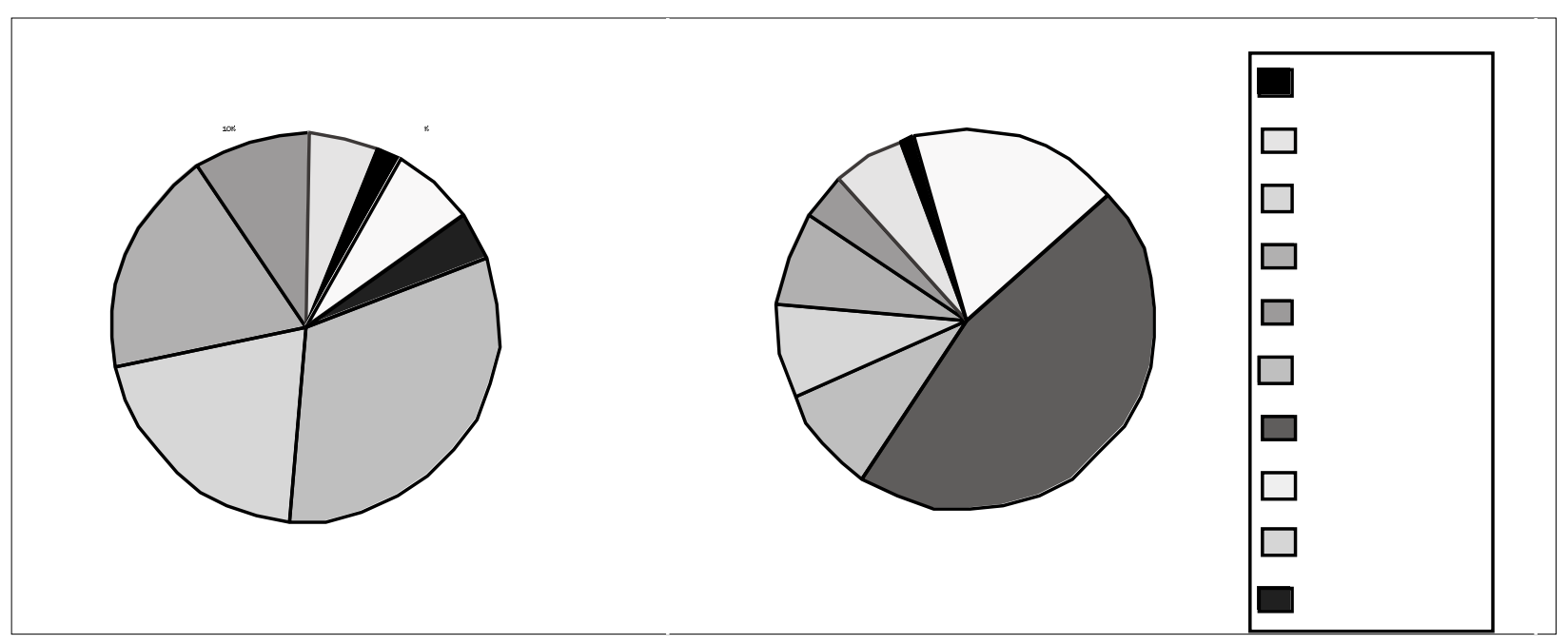

Figura 1

A. Nacionalidad de los padres inmigrantes. B. Lugar de nacimiento de los hijos de éstos. 
Tabla I

FACTORES EN RELACIÓN CON LA GESTACIÓN Y PARTO EN HIJOS DE INMIGRANTES E HIJOS DE POBLACION AUTOCTONA RECOGIDOS EN LAS HISTORIAS CLINICAS

\begin{tabular}{|c|c|c|c|c|}
\hline & & Hijos inmigrantes & $\begin{array}{l}\text { Hijos población } \\
\text { autóctona }\end{array}$ & $\begin{array}{l}\text { Significación } \\
\text { estadística }(p)\end{array}$ \\
\hline Tipo de parto & $\begin{array}{c}\text { Natural } \\
\text { Cesárea } \\
\text { Instrumental }\end{array}$ & $\begin{array}{c}73(76,04 \%) \\
16(16,67 \%) \\
7(7,29 \%)\end{array}$ & $\begin{array}{l}100(75,76 \%) \\
18(13,64 \%) \\
14(10,61 \%)\end{array}$ & $p=0,6(N S)$ \\
\hline Semana de gestación al nacer & $\begin{array}{l}\text { Pretérmino } \\
\text { A término } \\
\text { Provocado }\end{array}$ & $\begin{array}{c}1(1,05 \%) \\
94(98,95 \%) \\
0\end{array}$ & $\begin{array}{c}3(2,27 \%) \\
127(96,21 \%) \\
2(1,361,52 \%)\end{array}$ & No valorable \\
\hline $\begin{array}{l}\text { Peso del niño tras el } \\
\text { nacimiento }\end{array}$ & $\begin{array}{c}\text { Peso }<2.500 \mathrm{~g} \\
\text { Peso } 2.500-4.000 \mathrm{~g} \\
\text { Peso }>4.000 \mathrm{~g}\end{array}$ & $\begin{array}{c}2(2,32 \%) \\
70(81,40 \%) \\
14(16,28 \%)\end{array}$ & $\begin{array}{c}4(3,23 \%) \\
109(87,9 \%) \\
11(8,87 \%)\end{array}$ & No valorable \\
\hline $\begin{array}{l}\text { Ingreso del niño tras el } \\
\text { nacimiento }\end{array}$ & $\begin{array}{c}\text { Ingreso } \\
\text { No ingreso }\end{array}$ & $\begin{array}{l}17(18,08 \%) \\
77(81,92 \%)\end{array}$ & $\begin{array}{l}28(20,89 \%) \\
106(79,11 \%)\end{array}$ & $p=0,72$ (NS) \\
\hline
\end{tabular}

NS: no significativo

\section{Tabla II}

COMPARACIÓN DEL TIPO DE LACTANCIA RECIBIDA, CONSTANCIA DEL CALENDARIO VACUNAL O NO EN LAS HISTORIAS

Y ASISTENCIA A LAS CONSULTAS PROGRAMADAS, ENTRE HIJOS DE INMIGRANTES E HIJOS DE POBLACIÓN AUTÓCTONA

\begin{tabular}{lccc}
\hline & $\begin{array}{c}\text { Hijos de } \\
\text { inmigrantes }\end{array}$ & $\begin{array}{c}\text { Hijos de población } \\
\text { autóctona }\end{array}$ & $\begin{array}{c}\text { Significación } \\
\text { estadística (p) }\end{array}$ \\
\hline Lactancia materna & $87(87,88 \%)$ & $103(77,44 \%)$ & $p=0,06(\mathrm{CS})$ \\
Lactancia artificial & $12(12,12 \%)$ & $30(22,56 \%)$ & \\
Consta situación calendario vacunal & $104(70,48 \%)$ & $129(87,76 \%)$ & $p<0,05(\mathrm{~S})$ \\
No consta situación calendario vacunal & $43(29,52 \%)$ & $18(12,24 \%)$ & $\mathrm{p}<0,05(\mathrm{~S})$ \\
Ha faltado en alguna ocasión a consulta programada & $55(37,41 \%)$ & $25(17 \%)$ & $122(83 \%)$ \\
No ha faltado nunca a consulta programada & $92(62,59 \%)$ & 122 &
\end{tabular}

CS: Casi significativo. S: significativo.

observando una ausencia importante en las historias clínicas de los datos referidos a la gestación y parto, constancia de la situación del calendario vacunal e inicio o no de la lactancia materna en los niños HI.

La mayor duración de la lactancia materna observada en los niños HI podría atribuirse a la herencia de los respectivos países de origen, por lo general en vías de desarrollo, en los que bien por factores económicos o culturales cabría esperar una prolongación de la lactancia natural.

Un dato reseñable es el mayor incumplimiento de las consultas programadas por parte de la población inmigrante con respecto a la nacional. La posible inexistencia de programas de evaluación y se- guimiento del niño sano en sus países de procedencia podría explicarlo, estando este colectivo acostumbrado a acudir a los servicios sanitarios sólo ante la aparición de procesos agudos; aunque también podría deberse a la situación laboral de los padres, muchas veces tan inestable que les dificulta acudir a las consultas.

Si bien el número de consultas anuales resultó similar en ambas poblaciones, existen algunas diferencias en determinadas patologías. Llama la atención la diferencia encontrada en el caso de las gastroenteritis agudas que fueron un motivo de consulta más frecuente en los HA. Sería planteable si realmente esta enfermedad es más frecuente en estos niños o bien los padres inmigrantes no la con- 


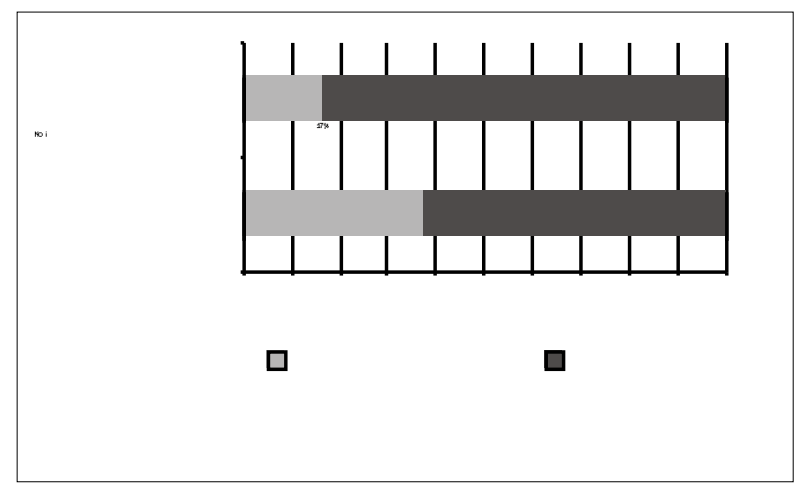

Figura 2

Relación de la asistencia a la consulta programada de los hijos de inmigrantes e hijos de población autóctona.

sideran motivo de consulta al pediatra por saber manejar estos cuadros, habituales en sus países de origen.

La mayor duración de la lactancia materna en HI podría explicar las diferencias encontradas en las otitis, más frecuentes en HA, dado el beneficio que produce la prolongación de la lactancia materna más allá de cuatro meses en la prevención de esta patología ${ }^{11}$.

El dolor abdominal inespecífico, sin embargo, fue más frecuente en HI. Creemos que esto podría atribuirse a cambios en la alimentación de este colectivo ${ }^{12,13}$ o bien a somatizaciones secundarias a la adaptación a una nueva realidad que supone el proceso de inmigración ${ }^{14}$.

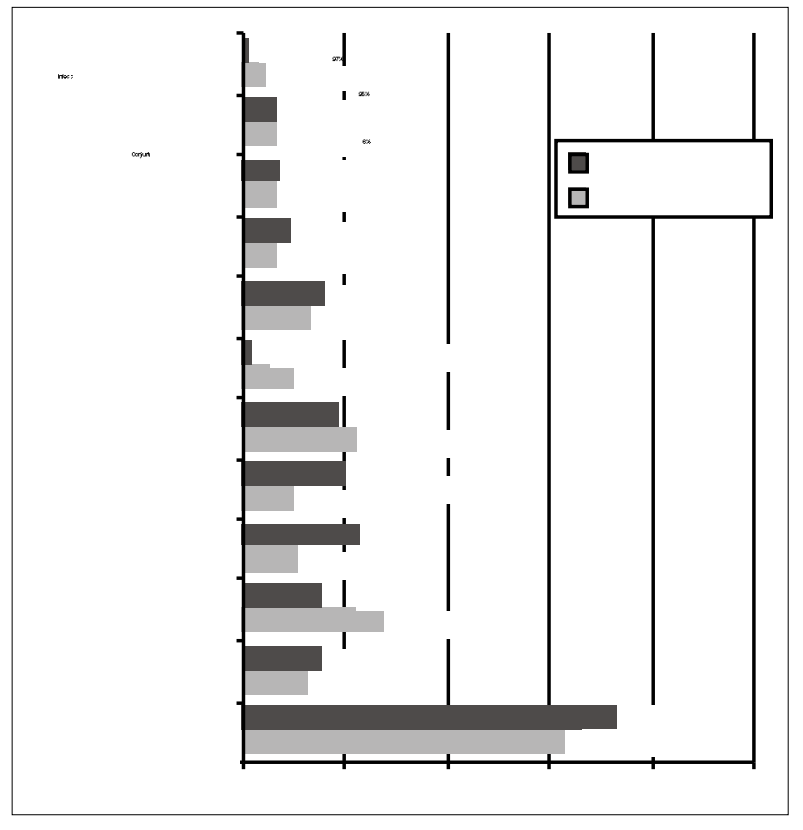

Porcentaje de niños (inmigrantes y no inmigrantes) que consultaron por diferentes patologías desde junio 2000 a junio 2001.
En cuanto a la solicitud de pruebas complementarias, es posible que el médico se vea condicionado por el origen de los niños y la posibilidad de que presenten patologías diferentes ${ }^{15-18}$. Ratifica esta opinión el hecho de haberse realizado varias pruebas de tuberculina en HI, siendo los resultados de todos ellos negativos. También se solicitaron un mayor número de cultivos de heces, a pesar de ser menor el número de consultas por gastroenteritis en esta población.

En definitiva, los hijos de inmigrantes se comportan de forma muy similar a los hijos de población autóctona en cuanto al número de consultas anuales y a las patologías más frecuentes observadas. Sin embargo, la diferencia encontrada en algunas patologías, el alto incumplimiento a consultas programadas y la mayor duración de la lactancia materna nos recuerda a los médicos de Atención Primaria que estamos ante una población con aún diferencias socioculturales.

Se le abre al médico de Atención Primaria un amplio campo de trabajo. El fenómeno migratorio, con todas sus consecuencias físicas y psicológicas, junto con la mezcla cultural que conlleva supone un desafío en el trabajo de la clínica diaria. Los pocos trabajos realizados hasta ahora que muestran el comportamiento sanitario de la población inmigrante, invitan a realizar nuevos estudios, que ahonden en la influencia de la inmigración en las posibles diferencias de comportamiento frente a la enfermedad, entre la población inmigrante y la autóctona, con lo que se

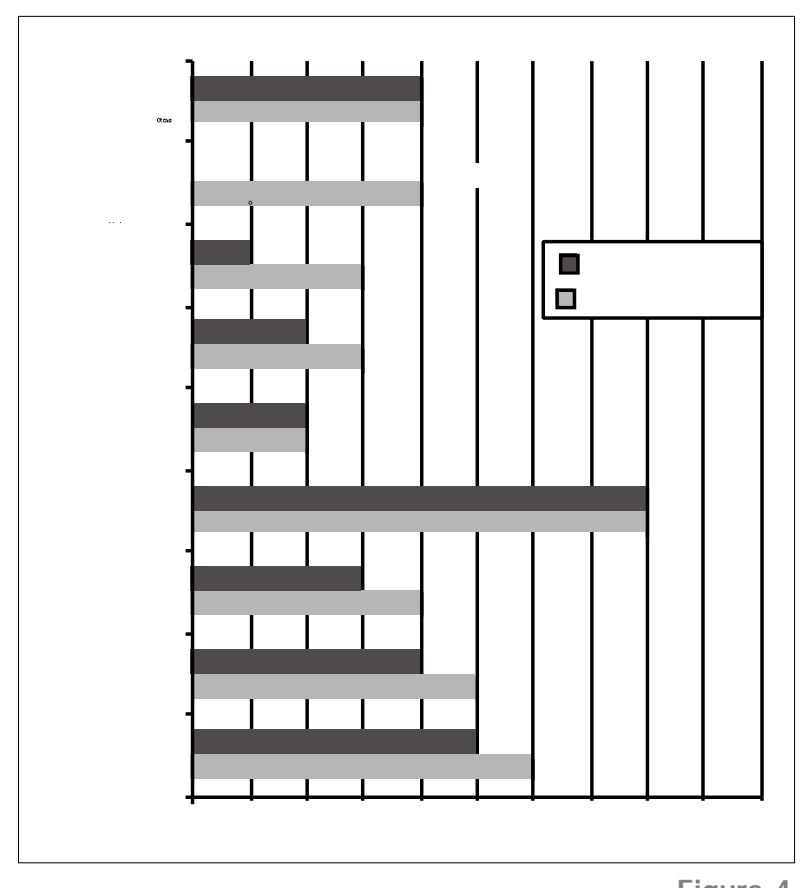

Figura 4

Pruebas complementarias solicitadas en ambas poblaciones desde junio 2000 a junio 2001. 
ayudaría al médico a afrontar esta nueva realidad social.

Creemos además, que el médico de Atención Primaria puede desempeñar desde su trabajo diario, una labor de promoción de la salud y educación sanitaria en este colectivo con el fin de lograr una completa integración en la sociedad.

\section{CORRESPONDENCIA}

Noemí Pérez Villaverde

Pza. Valdecaleros, $\mathrm{n}^{\circ} 12,3^{\circ} \mathrm{B}$

45002 Toledo

\section{Bibliografía}

1. Sanz B, Torres AM, Schumacher R. Características sociodemográficas y utilización de servicios sanitarios por la población inmigrante residente en un área de la Comunidad de Madrid. Aten Primaria 2000; 26: 314-8.

2. Bada JL. Repercusiones sociosanitarias de la inmigración en España. FMC 1996; 5: 277-8.

3. Del Rey J. Consideraciones epidemiológicas a la patología infecciosa emergente. El problema de las migraciones. An R Acad Nac Med (Madrid) 1997; 114 (1): 81-99.

4. Valtuena O. Problemas pediátricos de los hijos de inmigrantes en España. An R Acad Nac Med (Madrid) 2000; 117 (3): 607-16.

5. Jansá JM, Villalbí JR. La salud de lo inmigrantes y la atención primaria. Aten Primaria 1995; 15: 320-7.

6. Bruguera M, Sánchez JM. Hepatitis viral en población inmigrada y en niños adoptados un problema de magnitud desconocida en España. Med Clin (Barc) 2001; 0117: 595-6.

7. Lacalle M, Gil G, Sagardui JK, González E, Martínez R, Orden B. Resultados de la aplicación de un examen de salud en población inmigrante. Aten Primaria 2000; 25: 634-8.

8. Esteban MM. Motivos de consulta y características demográficas de una comunidad de inmigrantes "sin papeles" en al distrito de Usera-Villarverde (Madrid). Aten Primaria 2001; 27: 25-8.

9. Jaumà RM, Viñamata $\mathrm{B}$. Morbilidad atendida de la población inmigrante africana en un centro de salud. Aten Primaria $1994 ; 13: 283-9$.
10. Jansá JM. Inmigración extranjera en el Estado Español. Consideraciones desde la Salud Pública. Rev Esp Salud Pública 1998; 72: 165-8.

11. Olmo L, Lumbreras G, Lobo MA. Antibióticos en las infecciones respiratorias. FMC 2002; 9 (2): 119-35.

12. Montoya PP, Torres AM, Tortija ME. La alimentación de los inmigrantes marroquíes de la Comunidad de Madrid: factores que influyen en la selección de los alimentos. Aten Primaria 2001; 27: 264-70.

13. Olivan G. Assessing the health and nutritional status of illegal immigrant adolescents from Maghreb. An Esp Pediatr 2000; 53 (1): 17-20.

14. Roca C, Balanzó X, Fernández JL, Pujol E, Corachán M. Caracterización demográfica, motivos de consulta y morbilidad prevalente en la comunidad de inmigrantes africanos en la comarca del Maresme. Med Clin (Barc) 1999; 111: 215-7.

15. Caminero JA, Rodríguez F, Cuyas J. La inmigración en Canarias y su posible influencia sobre la frecuencia de tuberculosis. Med Clin (Barc) 2001; 116 (14): 557.

16. Balanzó X. Enfermedades importadas y medicina de la inmigración. Aten Primaria 1991; 8: 527-8.

17. López-Vélez R. Enfermedades infecciosa en inmigrantes. FMC 1996; 4: 222-8.

18. Rivas FP, Nácher M, Corrillero J, García-Herreros MT. Prevalencia de infección tuberculosa entre los inmigrantes magrebíes. Med Clin (Barc) 2000; 114: 245-9. 